[3] Tektonidou MG, Anapliotou M, Vlachoyiannopoulos P, Moutsopoulos HM. (2004) Presence of systemic autoimmune disorders in patients with autoimmune thyroid diseases. Ann Rheum Dis. 2004;63(9):1159-61. doi: 10.1136/ard.2004.022624.

Disclosure of Interest: None declared

DOI: 10.1136/annrheumdis-2017-eular.6752

\section{AB0475 CHANGES IN HEART RATE VARIABILITY REFLECT CHANGES IN CLINICAL STATUS AND PATIENT REPORTED OUTCOMES IN SYSTEMIC LUPUS ERYTHEMATOSUS: A LONGITUDINAL ANALYSIS}

A. Thanou ${ }^{1}$, S. Stavrakis ${ }^{2}$, J.C. Reynolds ${ }^{2}$, S. Kamp ${ }^{1}$, P. Kamp ${ }^{1}$, J.A. James ${ }^{1,2}$, J.T. Merrill ${ }^{1} .{ }^{1}$ Arthritis and Clinical Immunology Research Program, Oklahoma Medical Research Foundation; ${ }^{2}$ University of Oklahoma Health Sciences Center, Oklahoma City, United States

Background: We previously observed an association between systemic lupus erythematosus (SLE) disease activity and heart rate variability (HRV) with a preliminary observation of consistency between these two measures when disease activity changed between two timepoints (1)

Objectives: To prospectively test the hypothesis that HRV reflect longitudinal changes in clinical status and patient reported outcomes.

Methods: The current project evaluated HRV measurements using a 5 min ECG in SLE patients who completed a minimum of 2 visits in an ongoing clinical trial. HRV parameters were calculated in the time domain (RMSSD and pNN50) and the frequency domain [high frequency (HF) as well as low frequency to high frequency (LF/HF) ratio]. A mixed effects linear model, with generalized estimating equations to account for clustering of visits for each patient, was used to compare changes in HRV between paired visits and to examine linear associations between HRV parameters and clinical scores. All models were adjusted for baseline values of each HRV parameter.

Results: Forty nine patients (age 44.9 $11.7,46$ female), followed in a total of 413 paired visits (median time between visits 1 month), were included. Global BILAG score was inversely associated with RMSSD (regression coefficient $\beta=-1.39 \pm 0.01$; $p<0.0001)$. BILAG, SLEDAI and PGA were directly associated with the LF/HF ratio $(\beta=0.96 \pm 0.02 ; p<0.0001,0.42 \pm 0.10 ; p<0.0001$ and $0.83 \pm 0.09 ; p<0.0001$, respectively). Changes in BILAG were inversely associated with changes in RMSSD and $p N N 50$ ( $\beta=-7.0 \pm 1.9 ; p=0.003$ and $-1.6 \pm 0.04 ; p<0.0001$, respectively). BILAG changes were also directly associated with changes in the LF/HF ratio $(\beta=0.78 \pm 0.05 ; p<0.0001)$. Categorical improvement, defined as $\geq 1$ letter grade improvement in BILAG and no new BILAG A or B scores, occurred in $77(19 \%)$ visit pairs (group 1) and either no improvement or worsening in 335 (81\%) group 2. RMSSD and HF increased in group 1 compared to group 2 (group difference $=-$ $33.3 \pm 10.1 ; p=0.001$ and $-30.9 \pm 4.1 ; p<0.0001$, respectively), and the LF/HF ratio decreased in group 1 compared to group 2 (group difference $=3.1 \pm 0.8 ; p=0.002$ ). Changes in Physical Component Summary (PCS) of SF36v2 were inversely related to changes in SLEDAI and PGA $(\beta=-0.39 \pm 0.14 ; p=0.006$ and $-0.19 \pm 0.02$; $p<0.0001$, respectively). Changes in Mental Component Summary (MCS) were inversely related to changes in BILAG, SLEDAI and PGA $(\beta=-0.23 \pm 0.07 ; p=0.0001$, $-0.31 \pm 0.10 ; p=0.002$ and $-0.08 \pm 0.03 ; p=0.008$, respectively). PCS was related to $\mathrm{HF}(\beta=0.67 \pm 0.28, p=0.01)$ whereas MCS was inversely related to the LF/HF ratio $(\beta=-0.11 \pm 0.03, p=0.0001)$. Changes in PCS were related to changes in pNN50 $(\beta=0.21 \pm 0.05, p=0.0001)$ and changes in MCS were related to changes in HF $(\beta=1.57 \pm 0.18 ; p<0.0001)$.

Conclusions: Changes in HRV reflect changes in clinical status and patient reported outcomes in patients with SLE. These data suggest that HRV may be a simple non-invasive tool used to gage or predict clinical improvement in SLE. Further studies are warranted.

\section{References:}

[1] Thanou A, Stavrakis S, Dyer JW, Munroe ME, James JA, Merrill JT. Impact of heart rate variability, a marker for cardiac health, on lupus disease activity. Arthritis Res Ther. 2016 Sep 2;18:197.

Disclosure of Interest: None declared

DOI: 10.1136/annrheumdis-2017-eular.5425

\section{AB0476 INNER EAR INVOLVEMENT IN SYSTEMIC RHEUMATIC DISEASES}

G. Tsirves ${ }^{1}$, P.V. Voulgari ${ }^{2}$, D. Archimandriti ${ }^{2}$, E. Pelechas ${ }^{2}$, D. Asimakopoulos ${ }^{1}$, A.A. Drosos ${ }^{2} .{ }^{1}$ Otorhinolaryngology Clinic; ${ }^{2}$ Rheumatology Clinic, Department of Internal Medicine, Medical School, University of Ioannina, Ioannina, Greece

Background: Patients with systemic rheumatic diseases have increased prevalence of sensorineural hearing loss (SNHL). Detection of cochlin specific antibodies has been reported in patients with idiopatic sensorineural hearing loss. Interestingly, cochlin has been shown a stronger link to autoimmune hearing loss. Objectives: Objective of this cross-sectional study was to calculate the prevalence of SNHL in patients with systemic rheumatic diseases and to detect human cochlin antibodies in their sera.

Methods: This was a prospective study. Patients older that 18 year old who gave informed consent and fulfilled the criteria of American College of Rheumatology for rheumatoid arthritis (RA), systemic lupus erythematosus (SLE), Sjogren's syndrome (SS) and systemic sclerosis (SSc) were included. Complete head and neck clinical examination was performed, including otoscopy, nasendoscopy and hearing investigation with pure tone audiometry $(250 \mathrm{~Hz}-8000 \mathrm{~Hz})$. All medical treatments as well as Disease activity score (DAS) 28 for RA and SLE disease activity index (SLEDAI) for SLE were documented. An average tone loss was calculated, taking as a starting point the loss in $\mathrm{dB}$ at various frequencies according the American Committee on Hearing and Equilibrium Guidelines for Meniere's disease and also recommendation 02/1 of "Bureau International d' Audiophonologie" (BIAP). Blood samples of the patients were tested for the presence of $\operatorname{lgG}$ anti-cochline antibodies (COCH-lgG). The results were compared with those of sex and age-matched healthy subjects

Results: We studied 133 patients (60 with RA, 41 with SLE, 24 with SS and 8 with SSc) and 133 healthy subjects. $61.4 \%$ of patients reported vertigo, $41 \%$ hyperacousis, $39 \%$ hearing loss, $38 \%$ tinnitus, $37.9 \%$ headache and $2.1 \%$ sensation of ear pressure with unremarkable otoscopy. The prevalence of SNL was increased in patients affected by RA, SLE, SS and SSc in comparison to healthy controls $(66.6 \%, 31.71 \%, 54.17 \%$ and $75 \%$ respectively). The average hearing thresholds (AHT) calculated using BIAP recommendation 02/1 were significantly increased in RA compared to SLE. AHT were also increased in patients with RA and secondary SS but without statistical significance compared to RA patients. There was a statistically significant correlation between AHT and DAS28 in RA. No correlation observed between AHT and SLEDAI. COCH-IgG were detected in two samples (one patient with RA and one with RA and SS).

Conclusions: Cochlin has been shown to have a stronger link to autoimmune hearing loss, but our study concluded no correlation of hearing loss with human cochlin IgG $(\mathrm{COCH})$. Additional prospective studies are needed to elucidate its pathogenesis.

Disclosure of Interest: None declared

DOI: 10.1136/annrheumdis-2017-eular.4805

\section{AB0477 VITAMIN D STATUS, SYSTEMIC LUPUS ERYTHEMATOSUS ACTIVITY AND ENDOTHELIAL DYSFUNCTION}

A. Caraba, V. Crisan. Internal Medicine-Rheumatology, University of Medicine and Pharmacy "Victor Babes" Timisoara, Romania, Timisoara, Romania

Background: Systemic lupus erythematosus (SLE) is an autoimmune inflammatory disease, in which cardiovascular complications due to premature and accelerated atherosclerosis represent a serious problem. Endothelial dysfunction is the first step in the atherosclerosis development. Low levels of vitamin D have a high prevalence in lupus patients, correlating with high prevalence of cardiovascular diseases in these patients.

Objectives: The aim of this study was represented by the assessment of endothelial dysfunction in patients with active systemic lupus erythematosus, and correlations of this with SLE activity and vitamin D status.

Methods: The study was performed on a group of 35 female patients with active systemic lupus erythematosus. In all the patients were assessed: SLE activity using SLEDAI (SLE Disease Activity Index), vitamin D status and endothelial dysfunction by means of flow-mediated dilation (FMD). Data were presented as mean \pm standard deviation. The statistically analysis was performed using Pearson's test, $p<0.05$ was considered statistically significant.

Results: The mean age of the studied patients was $33.68 \pm 8.55$ years, and the average duration of SLE was $8.91 \pm 5.29$ years. The assessed parameters had the values: SLEDAI $9.02 \pm 4.51$, vitamin D $12.79 \pm 2.45 \mu \mathrm{g} / \mathrm{l}$ (4 patients had vitamin D deficiency, and 31 patients had vitamin D insufficiency), and FMD $9.26 \pm 2.34 \%$. There were significant correlations between vitamin D levels and FMD $(r=0.4517$, $p=0.003)$, and between vitamin D levels and SLEDAI ( $r=-0.6297, p=0.00025)$.

Conclusions: Low values of vitamin $D$ levels were present in all the studied patients, correlating with the systemic lupus erythematosus activity, and endothelial dysfunction.

References:

[1] Reynolds JA, Bruce IN. Vitamin D in systemic lupus erythematosus: potential beyond bone health. Int J Clin Rheumatol 2009; 4(3): 297-309.

[2] Emam FE, Abd El-Wahab TM, Mohammed MS, et al. Assessment of serum vitamin D level in patients with systemic lupus erythematosus. Egypt Rheumatol Rehabil 2014; 41:71-78.

Disclosure of Interest: None declared

DOI: 10.1136/annrheumdis-2017-eular.3282

\section{AB0478 COMPARATIVE STUDY BETWEEN PATIENTS WITH NORMAL AND OVERWEIGHT IN A COHORT OF SYSTEMIC LUPUS ERITEMATHOSUS FROM ARGENTINA}

A.M. Beron, S. Sapag Duran, M. Garcia Carrasco, A.M. Sapag Duran, G. Medina, G. Nasswetter, D. Dubinsky. Rheumatology, Clinical Hospital "Jose de San Martin", University of Buenos Aires., CABA, Argentina

Background: Systemic lupus erythematous (SLE) have an increase cardiovascular risk, worsened by overweight and obesity. Increased BMI is associated with other severe complications and comorbidities as lupus nephritis, hypertension, insulin resistance and dyslipemia. Body weight is a modifiable risk factor. Objectives: To determine the frequency of overweight and obesity in patients with SLE and their impact on this disease 